\title{
Effects of Nrf2 knockdown on the properties of irradiated cell conditioned medium from A549 human lung cancer cells
}

\author{
HIRONORI YOSHINO $^{1}$, KANNA MURAKAMI ${ }^{2}$, MIKOTO NAWAMAKI $^{2}$ and IKUO KASHIWAKURA ${ }^{1}$ \\ ${ }^{1}$ Department of Radiation Science, Hirosaki University Graduate School of Health Sciences; ${ }^{2}$ Department of \\ Radiological Technology, Hirosaki University School of Health Sciences, Hirosaki, Aomori 036-8564, Japan
}

Received February 22, 2018; Accepted March 7, 2018

DOI: $10.3892 /$ br.2018.1073

\begin{abstract}
The nuclear factor erythroid 2-related factor 2 (Nrf2) plays an important role in cellular defense against oxidative stress. Recent studies have demonstrated that Nrf2 is a useful target for cancer treatment, including radiation therapy. Ionizing radiation affects, not only the irradiated cells, but also the non-irradiated neighboring cells, and this effect is known as radiation-induced bystander effect. Upon exposure to radiation, the irradiated cells transmit signals to the non-irradiated cells via gap junctions or soluble factors. These signals in turn cause biological effects, such as a decrease in the clonogenic potential and cell death, in the non-irradiated neighboring cells. Nrf2 inhibition enhances cellular radiosensitivity. However, whether this modification of radiosensitivity by Nrf2 inhibition affects the radiation-induced bystander effects is unknown. In this study, we prepared an Nrf2 knockdown human lung cancer cell A549 and investigated whether the effects of irradiated cell conditioned medium (ICCM) on cell growth and cell death induction of non-irradiated cells vary depending on the Nrf2 knockdown. We found that Nrf2 knockdown resulted in a decrease in the cell growth and an increase in the radiosensitivity of A549 cells. When non-irradiated A549 cells were transfected with control siRNA and treated with ICCM, no significant difference was observed in the cell growth and proportion of Annexin $\mathrm{V}^{+}$dead cells between ICCM from non-irradiated cells and that from 2 or 8 Gy-irradiated cells. Similarly, no significant difference was observed in the cell
\end{abstract}

Correspondence to: Dr Hironori Yoshino, Department of Radiation Science, Hirosaki University Graduate School of Health Sciences, 66-1 Hon-cho, Hirosaki, Aomori 036-8564, Japan

E-mail: hyoshino@hirosaki-u.ac.jp

Abbreviations: Nrf2, nuclear factor erythroid 2-related factor 2; ICCM, irradiated cell conditioned medium; ROS, reactive oxygen species; PI, propidium iodide; HRP, horseradish peroxidase; SDS-PAGE, sodium dodecyl sulfate-polyacrylamide gel electrophoresis; SD, standard deviation

Key words: Nrf2, ionizing radiation, bystander effect, radiosensitivity, irradiated cell conditioned medium growth and cell death induction upon treatment with ICCM in the Nrf2 knockdown A549 cells. Taken together, these results suggest that Nrf2 knockdown decreases cell growth and enhances the radiosensitivity of A549 cells; however, it does not alter the effect of ICCM on cell growth.

\section{Introduction}

Ionizing radiation causes biological effects, such as cell death and chromosomal aberrations, on cells. There are many evidences that ionizing radiation affects, not only the irradiated cells, but also the non-irradiated neighboring cells (1-3). Such response is known as radiation-induced non-targeted effects, which includes genomic instability and radiation-induced bystander effects. Genomic instability is characterized by effects such as delayed gene mutation and chromosomal aberrations that occur in the progeny of irradiated cells (3). In radiation-induced bystander effects, it has been suggested that the irradiated cells transmit signals to the non-irradiated cells via gap junctions or soluble factors (such as cytokines and growth factors) $(1,2)$. To investigate the soluble factor-mediated bystander effects in vitro, non-irradiated cells were co-cultured with irradiated cells or cultured in irradiated cell conditioned medium (ICCM). It has been reported that non-irradiated cells co-cultured with irradiated cells or treated with ICCM undergo various biological responses, such as DNA double-strand breaks, decrease in clonogenic cell survival, and cell death, similar to irradiated cells (1).

The nuclear factor erythroid 2-related factor 2 (Nrf2) is a transcription factor, which plays an important role in cellular defense against oxidative stress (4). In response to oxidative stresses, such as reactive oxygen species (ROS), Nrf2 rapidly translocates to the nucleus and induces the expression of various antioxidant genes, such as heme oxygenase-1. Recent studies have demonstrated the over-activation of Nrf2 by the somatic mutation of $\mathrm{Nrf} 2$ or its inhibitor Keapl in various types of cancer $(5,6)$. For example, Nrf2 promotes the proliferation and metastasis of lung cancer and oesophageal cancer cells $(7,8)$. Moreover, the over-activation of Nrf2 leads to resistance toward chemotherapeutic agents $(7,9)$.

Low linear energy transfer radiations, such as X-rays, cause biological damage through ROS production $(10,11)$. Nrf2-mediated cellular defense is involved in the cellular response to ionizing radiation (12-14). Furthermore, it has 
been reported that Nrf2 downregulation by shRNA and its inhibition using a small molecular weight compound 4-(2-cyclohexylethoxy)aniline enhance the sensitivity to ionizing radiation $(15,16)$. These results indicate that Nrf2 is a useful target to improve the efficacy of cancer radiotherapy. However, it remains unknown whether a modification of the radiosensitivity by $\mathrm{Nrf} 2$ knockdown affects the property of ICCM.

In this study, we hypothesized than the upregulation of radiosensitivity by Nrf2 inhibition alters the ICCM-mediated effects on non-irradiated cells. To test this hypothesis, we transfected siRNA against Nrf2 into A549 human lung cancer cells, which constitutively overexpress Nrf2 because they have a somatic mutation in Keapl (5). We then investigated whether the effects of ICCM from A549 cells on cell growth and cell death induction vary depending on the Nrf2 knockdown.

\section{Materials and methods}

Reagents. Propidium iodide (PI) was purchased from Sigma-Aldrich (St. Louis, MO, USA). Anti-Nrf2 antibody (cat. no. sc-13032) was purchased from Santa Cruz Biotechnology, Inc. (Santa Cruz, CA, USA). Anti- $\beta$-actin antibody (cat. no. 4967) and anti-rabbit horseradish peroxidase (HRP)-linked IgG antibody (cat. no. 7074) were purchased from Cell Signaling Technology Japan, K.K. (Tokyo, Japan). Ambion's Silencer ${ }^{\circledR}$ Select Pre-designed siRNA against the gene encoding Nrf2 (ID: s9492) and Silencer ${ }^{\circledR}$ Select Negative Control 1 siRNA were purchased from Life Technologies Corporation; Thermo Fisher Scientific, Inc. (Waltham, MA, USA).

Cell culture. The A549 lung cancer cell line was purchased from the American Type Culture Collection (Manassas, VA, USA). A549 cells were maintained at $37^{\circ} \mathrm{C}$ in a humidified $5 \% \mathrm{CO}_{2}$ atmosphere and cultured in RPMI 1640 medium (Gibco; Thermo Fisher Scientific, Inc.) supplemented with $1 \%$ penicillin/streptomycin (Gibco; Thermo Fisher Scientific, Inc.) and 10\% heat-inactivated FBS (Japan Bioserum Co., Ltd., Nagoya, Japan).

siRNA transfection. A549 cells were transfected with target or control siRNA using Lipofectamine RNAiMAX (Invitrogen; Thermo Fisher Scientific, Inc.), as previously reported (17). The final concentration of siRNAs in the medium was $10 \mathrm{nM}$. After incubating the cells with the medium containing siRNAs for $48 \mathrm{~h}$, transfected cells were collected and used for subsequent analyses.

In vitro irradiation. The cells were irradiated $(150 \mathrm{kVp}$, $20 \mathrm{~mA}, 0.5-\mathrm{mm} \mathrm{Al}$ and $0.3-\mathrm{mm} \mathrm{Cu}$ filters) using an X-ray generator (MBR-1520R-3; Hitachi Medical Corporation, Tokyo, Japan) at a distance of $45 \mathrm{~cm}$ from the focus and a dose rate of 1.00-1.05 $\mathrm{Gy} / \mathrm{min}$.

Clonogenic survival assay. To examine the radiosensitivity, the cells were seeded on 60-mm diameter culture dishes (Iwaki, Chiba, Japan) and cultured overnight. After culturing for $6 \mathrm{~h}$, the cells were exposed to X-ray radiation and incubated for the next 8-11 days. Next, the cells were fixed with methanol and stained with Giemsa solution (Wako Pure Chemical Industries, Ltd., Osaka, Japan). Colonies containing >50 cells were counted.

Sodium dodecyl sulfate-polyacrylamide gel electrophoresis(SDS-PAGE) andwesternblotanalysis.Protein preparation and determination of the protein concentration were performed as reported previously (18). SDS-PAGE and western blot analysis were performed as reported previously (19). The following concentrations of primary antibodies were used: Anti-Nrf2 antibody (dilution, 1:3,000) and anti-actin antibody (dilution, 1:4,000). The secondary antibody used was HRP-linked anti-rabbit IgG antibody (dilution, 1:10,000). Antigens were visualized using the ECL Prime Western Blotting Detection System (GE Healthcare Life Sciences, Chalfont, UK). Blots were stripped using a commercially available stripping solution (Wako Pure Chemical Industries, Ltd.).

Medium transfer experiments. The schematic for medium transfer experiments is shown in Fig. 1. Approximately $2.4 \times 10^{5}$ transfected cells were seeded onto $35-\mathrm{mm}$ culture dishes and cultured for $5 \mathrm{~h}$ to promote their adherence to the dish. The cells were then exposed to X-rays, cultured for $24 \mathrm{~h}$, and the cell conditioned medium was then collected by centrifugation (1,000 rpm for $5 \mathrm{~min}$ at room temperature). After centrifugation, the supernatant was collected and filtered using a $0.45-\mathrm{mm}$ syringe filter (2053-025; Iwaki) to remove cells and debris. The filtrated cell conditioned medium (hereafter referred to as ICCM) was used for subsequent experiments.

One day before collecting the ICCM, approximately $6.0 \times 10^{4}$ cells were seeded onto $35-\mathrm{mm}$ culture dishes for cell death analysis, or in a 12-well plate (BD Falcon) (100 or 120 cells) for colony assay, and cultured overnight to allow for their adherence to the dish. On the following day, the medium was aspirated and ICCM was added to the $35-\mathrm{mm}$ culture dishes or to the 12-well plate. After 3 days of culturing, the cells that were seeded in the $35-\mathrm{mm}$ culture dishes were collected using $0.1 \%$ trypsin-ethylene diamine tetraacetic acid (Gibco; Thermo Fisher Scientific, Inc.), and the number of viable cells was counted using the trypan blue dye exclusion assay. Finally, the harvested cells were used to perform the cell death analysis.

The cells that were seeded in the 12-well plates for colony assay were incubated for 8-10 days. The cells were then fixed with methanol and stained with Giemsa solution. Colonies containing $>50$ cells were counted.

Cell death analysis. Cell death was analyzed using Annexin V-FITC (BioLegend, Inc., San Diego, CA, USA), PI, and Annexin V binding buffer (BioLegend, Inc.), as reported previously (20). Stained cells were analyzed by performing flow cytometry (Cytomics FC500; Beckman Coulter, Inc., Brea, CA, USA). In the Annexin V/PI quadrant gating, Annexin $\mathrm{V}^{-} / \mathrm{PI}^{-}$, Annexin $\mathrm{V}^{+} / \mathrm{PI}^{-}$, and Annexin $\mathrm{V}^{+} / \mathrm{PI}^{+}$were used to identify the fraction of viable cells, early apoptotic cells, and late apoptotic/necrotic cells, respectively.

Statistical analysis. Data are presented as mean \pm standard deviation. Comparisons between control and experimental groups were performed using a two-sided Student's t-test or 


\section{A}
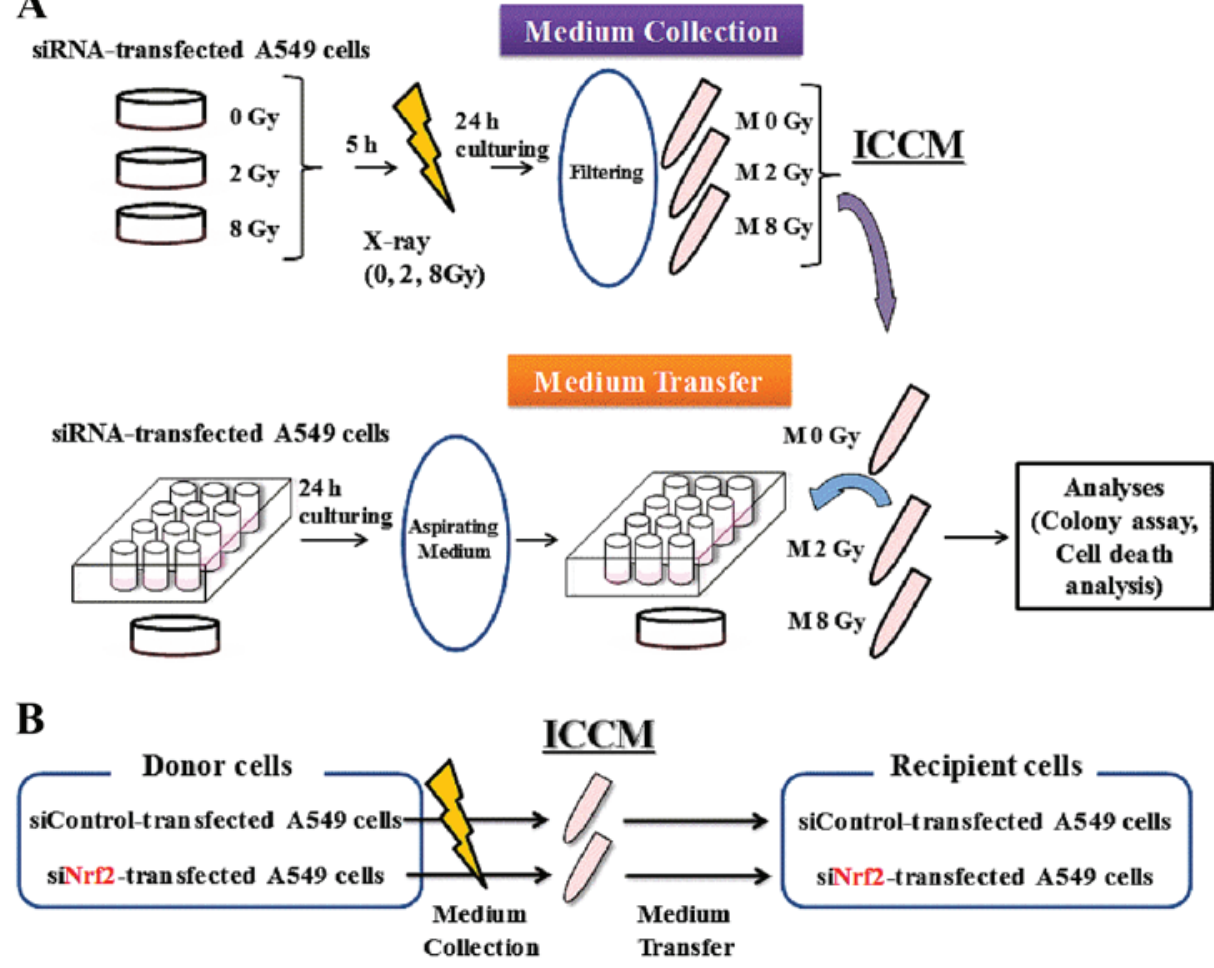

Figure 1. Schematic for medium transfer experiments. (A) The procedures of the collection of irradiated cell conditioned medium and treatment with ICCM. (B) The relationship between ICCM donor and recipient cells. ICCM, irradiated cell conditioned medium; Nrf2, nuclear factor erythroid 2-related factor 2.

a two-sided Mann-Whitney U-test depending on the data distribution. Non-parametric multiple data were analyzed using the Kruskal-Wallis test followed by the Steel test. Differences were considered significant at $\mathrm{P}<0.05$. All statistical analyses were performed using Excel 2016 software (Microsoft Corporation, Redmond, WA, USA), with an add-on software Statcel 4 (The Publisher OMS Ltd., Tokyo, Japan).

\section{Results and Discussion}

We investigated whether the effects of ICCM from A549 cells on growth and death induction vary depending on the regulation of radiosensitivity by Nrf2 knockdown.

To this aim, we first transfected A549 cells with siRNA against Nrf2, which led to a decreased Nrf2 protein expression in these cells (Fig. 2A). Furthermore, the radiosensitivity of the Nrf2 knockdown cells was significantly higher than that of the control cells (Fig. 2B). Consistent with previous reports (14-16), our results indicate that Nrf2 regulates the radiosensitivity of cancer cells.

Next, we investigated the effects of ICCM on the growth and death induction in non-irradiated cells. The relationship between ICCM donor and recipient cells is shown in Fig. 1B. When non-irradiated A549 cells that were transfected with control siRNA were treated with ICCM, no significant difference was observed in the cell growth (estimated using the trypan blue dye exclusion and colony assays) between ICCM from non-irradiated cells and that from 2 or 8 Gy-irradiated cells (Fig. 3A and B). Furthermore, we did not observe any significant difference in the proportion of Annexin $\mathrm{V}^{+}$dead cells upon treatment with ICCM (Fig. 3C). Next, we performed

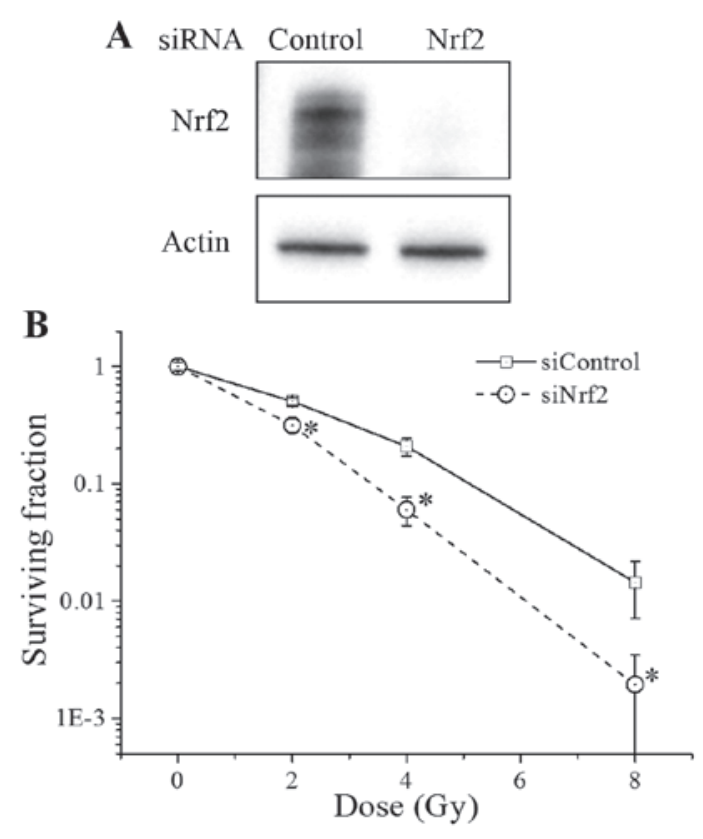

Figure 2. Effect of Nrf2 knockdown on the expression of Nrf2 protein and radiosensitivity of A549 cells. (A) A549 cells treated with control siRNA or siRNA against Nrf2 were harvested for western blotting of Nrf2, and actin was used as a loading control. Representative blots are shown. (B) Cellular radiosensitivity of Nrf2 knockdown A549 cells was analyzed by performing the clonogenic survival assay. Data are presented as mean \pm standard deviation of three independent experiments performed in triplicate. ${ }^{*} \mathrm{P}<0.01$ compared with control cells. Nrf2, nuclear factor erythroid 2-related factor 2.

similar experiments using the Nrf2 knockdown A549 cells and found that the cell growth and clonogenic potential were significantly lower for Nrf2 knockdown cells treated with 
A

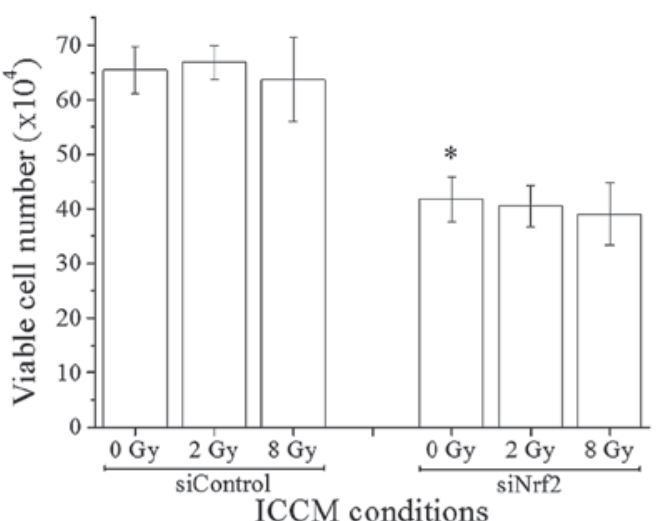

C
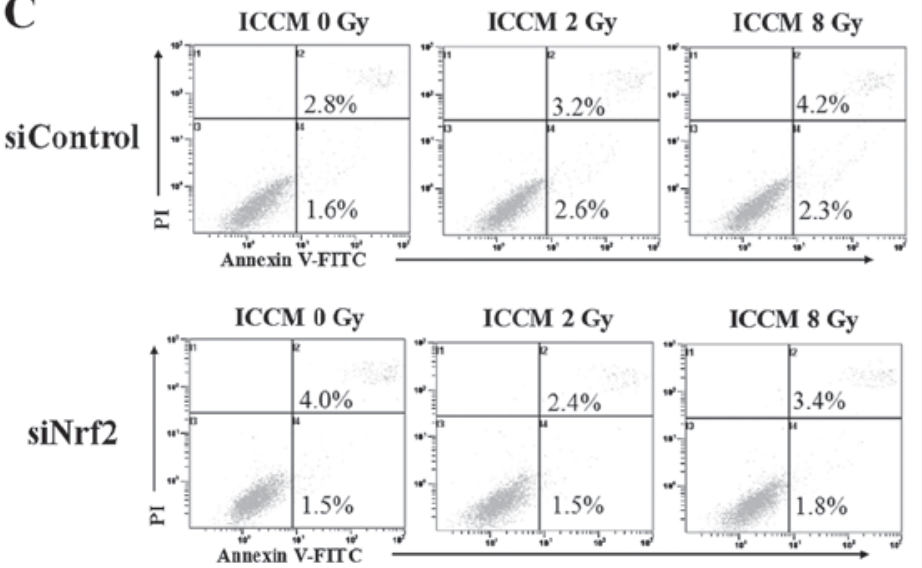

B
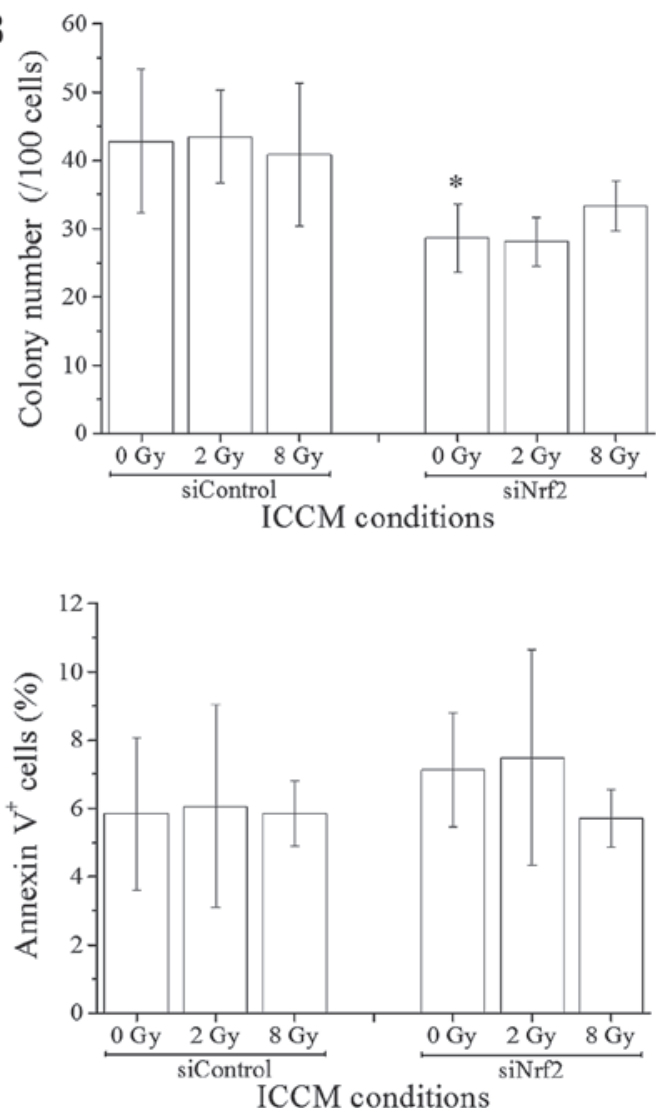

Figure 3. Cytotoxic effects of irradiated cell conditioned medium from Nrf2 knockdown A549 cells. (A) Non-irradiated control A549 or Nrf2 knockdown A549 cells were cultured in the presence of ICCM. After 3 days of culturing, the cells were harvested and the number of viable cells was counted using the trypan blue exclusion assay. Data are presented as mean $\pm \mathrm{SD}$ of three independent experiments. ${ }^{*} \mathrm{P}<0.01$ compared with siRNA control non-irradiated cells. (B) To investigate the clonogenic potential, the cells were cultured in the presence of ICCM for 8-10 days. After culturing, the number of cell colonies was counted. Data are presented as mean $\pm \mathrm{SD}$ of three independent experiments performed in triplicate; ${ }^{*} \mathrm{P}<0.01$ compared with siRNA control non-irradiated cells. (C) The cells were cultured in the presence of ICCM. After 3 days of culturing, the cells were harvested for cell death analysis. Annexin V/PI staining was performed to evaluate cell death. Left panel: Representative histograms of Annexin V/PI staining, with inset numbers indicating the percentage of Annexin $\mathrm{V}^{+} / \mathrm{PI}^{-}$and Annexin $\mathrm{V}^{+} / \mathrm{PI}^{+}$cells. Right panel: Percentages of Annexin $\mathrm{V}^{+}$cells are presented as mean $\pm \mathrm{SD}$ of three independent experiments. Nrf2, nuclear factor erythroid 2-related factor 2; ICCM, irradiated cell conditioned medium; SD, standard deviation.

non-irradiated Nrf2 knockdown cell conditioned medium than for those treated with control siRNA (Fig. 3A and B). When the medium transfer experiments were performed using ICCM from Nrf2 knockdown cells, no significant difference was observed in the cell growth, clonogenic potential, and proportion of Annexin $\mathrm{V}^{+}$dead cells (Fig. 3A-C). Taken together, these results suggest that although Nrf2 knockdown affects the cell growth of A549 cells, it does not alter the effects of ICCM on the cell growth and cell death induction. Yang et al have reported that the cell conditioned medium from irradiated A549 cells causes cytotoxicity in the non-irradiated A549 cells (21); however, we could not observe the cytotoxic effects of ICCM from A549 cells. It has been reported that bystander effects by photon-irradiation are strongly influenced by radiation dose (1). While we used ICCM from A549 cells exposed to 2 or 8 Gy X-ray, Yang et al used ICCM from A549 cells exposed to 0.5-2 Gy X-ray. However, because ICCM from 2 Gy irradiation decreased the clonogenic cell survival of non-irradiated cells (21), it seems that the discrepancy between our results and their results is due to another factor and not the radiation dose. Suzuki has reported that the decrease in clonogenic cell survival caused by bystander effects varies depending on the radiation quality, such as the types of ions (22). While Yang et al used $6 \mathrm{MV}$ $\mathrm{X}$-ray radiation for their study, we used $150 \mathrm{kVp} \mathrm{X}$-ray (21). Thus, the difference in the energy of X-ray likely explains the discrepancy between our results and those of Yang et al (21).

Howe et al have previously investigated the relationship between intrinsic radiosensitivity and bystander effects (23). They treated HaCaT human skin cells with ICCM from irradiated lymphocytes obtained from healthy individuals and colorectal carcinoma patients and investigated the bystander effects of ICCM using a viability test. They also examined the intrinsic radiosensitivity of the irradiated lymphocytes and investigated the relationship between radiosensitivity and bystander effects. They found no significant relationship between the radiation-induced intrinsic sensitivity and bystander effects. In line with their report, the modification of radiosensitivity by Nrf2 knockdown did not alter the effects of ICCM on cell growth and cell death induction in our study. Therefore, it is unlikely that cellular radiosensitivity determines the cytotoxic effects of ICCM.

In conclusion, the present findings suggest that $\mathrm{Nrf} 2$ knockdown enhanced the radiosensitivity of A549 cells, but it did not alter the effects of ICCM on cell growth. It is concerning that the radiation-induced non-targeted effects, 
such as chromosomal aberrations, are related to the risk of carcinogenesis in normal cells. Therefore, considering combined therapy comprising Nrf2-targeted cancer therapy and radiation therapy, future studies to investigate whether the radiation-induced bystander effects against normal cells vary depending on Nrf2 inhibition knockdown are needed.

\section{Acknowledgements}

The authors would like to thank Enago (www.enago.jp) for the English language review.

\section{Funding}

This study was supported by JSPS KAKENHI (grant no. JP15K09985).

\section{Availability of data and material}

All data generated or analyzed in this study are included in this article.

\section{Authors' contributions}

HY initiated the research. HY, KM, and MN performed experiments, collected data, and analyzed data. HY and IK wrote, reviewed, and revised manuscript. All authors read and approved the final manuscript.

\section{Ethics approval and consent to participate}

Not applicable.

\section{Consent for publication}

Not applicable.

\section{Competing interests}

The authors declare that there are no conflicts of interest regarding the publication of this study.

\section{References}

1. Tomita M and Maeda M: Mechanisms and biological importance of photon-induced bystander responses: Do they have an impact on low-dose radiation responses. J Radiat Res (Tokyo) 56: 205-219, 2015.

2. Hamada N, Maeda M, Otsuka K and Tomita M: Signaling pathways underpinning the manifestations of ionizing radiation-induced bystander effects. Curr Mol Pharmacol 4: 79-95, 2011.

3. Kadhim MA and Hill MA: Non-targeted effects of radiation exposure: Recent advances and implications. Radiat Prot Dosimetry 166: 118-124, 2015.

4. Itoh K, Tong KI and Yamamoto M: Molecular mechanism activating Nrf2-Keapl pathway in regulation of adaptive response to electrophiles. Free Radic Biol Med 36: 1208-1213, 2004.
5. Singh A, Misra V, Thimmulappa RK, Lee H, Ames S, Hoque MO, Herman JG, Baylin SB, Sidransky D, Gabrielson E, et al: Dysfunctional KEAP1-NRF2 interaction in non-small-cell lung cancer. PLoS Med 3: e420, 2006.

6. Na HK and Surh YJ: Oncogenic potential of Nrf2 and its principal target protein heme oxygenase-1. Free Radic Biol Med 67: 353-365, 2014.

7. Ohta T, Iijima K, Miyamoto M, Nakahara I, Tanaka H, Ohtsuji M, Suzuki T, Kobayashi A, Yokota J, Sakiyama T, et al: Loss of Keap1 function activates Nrf2 and provides advantages for lung cancer cell growth. Cancer Res 68: 1303-1309, 2008.

8. Kitano Y, Baba Y, Nakagawa S, Miyake K, Iwatsuki M, Ishimoto T, Yamashita YI, Yoshida N, Watanabe M, Nakao M and Baba H: Nrf2 promotes oesophageal cancer cell proliferation via metabolic reprogramming and detoxification of reactive oxygen species. J Pathol 244: 346-357, 2018.

9. Zhong Y, Zhang F, Sun Z, Zhou W, Li ZY, You QD, Guo QL and $\mathrm{Hu}$ R: Drug resistance associates with activation of Nrf2 in MCF-7/DOX cells, and wogonin reverses it by down-regulating Nrf2-mediated cellular defense response. Mol Carcinog 52: 824-834, 2013.

10. Mikkelsen RB and Wardman P: Biological chemistry of reactive oxygen and nitrogen and radiation-induced signal transduction mechanisms. Oncogene 22: 5734-5754, 2003.

11. Lee SY, Jeong EK, Ju MK, Jeon HM, Kim MY, Kim CH, Park HG, Han SI and Kang HS: Induction of metastasis, cancer stem cell phenotype, and oncogenic metabolism in cancer cells by ionizing radiation. Mol Cancer 16: 10, 2017.

12. Tsukimoto M, Tamaishi N, Homma T and Kojima S: Low-dose gamma-ray irradiation induces translocation of Nrf2 into nuclear in mouse macrophage RAW264.7 cells. J Radiat Res (Tokyo) 51: 349-353, 2010.

13. McDonald JT, Kim K, Norris AJ, Vlashi E, Phillips TM, Lagadec C, Della Donna L, Ratikan J, Szelag H, Hlatky L and McBride WH: Ionizing radiation activates the Nrf2 antioxidant response. Cancer Res 70: 8886-8895, 2010.

14. Yoshino H, Kiminarita T, Matsushita Y and Kashiwakura I: Response of the Nrf2 protection system in human monocytic cells after ionising irradiation. Radiat Prot Dosimetry 152: 104-108, 2012.

15. Singh A, Bodas M, Wakabayashi N, Bunz F and Biswal S: Gain of Nrf2 function in non-small-cell lung cancer cells confers radioresistance. Antioxid Redox Signal 13: 1627-1637, 2010.

16. Lee S, Lim MJ, Kim MH, Yu CH, Yun YS, Ahn J and Song JY: An effective strategy for increasing the radiosensitivity of human lung cancer cells by blocking Nrf2-dependent antioxidant responses. Free Radic Biol Med 53: 807-816, 2012.

17. Yoshino H, Iwabuchi M, Kazama Y and Kashiwakura I: Effects of retinoic acid-inducible gene-I-like receptors activations and ionizing radiation cotreatment on cytotoxicity against human non-small cell lung cancer in vitro. Oncol Lett 15:4697-4705, 2018.

18. Yoshino H, Kumai Y and Kashiwakura I: Effects of endoplasmic reticulum stress on apoptosis induction in radioresistant macrophages. Mol Med Rep 15: 2867-2872, 2017.

19. Yoshino H, Saitoh T, Kozakai M and Kashiwakura I: Effects of ionizing radiation on retinoic acid-inducible gene-I-like receptors. Biomed Rep 3: 59-62, 2015.

20. Fukushi S, Yoshino H, Yoshizawa A and Kashiwakura I: p53-independent structure-activity relationships of 3-ring mesogenic compounds' activity as cytotoxic effects against human non-small cell lung cancer lines. BMC Cancer 16: 521, 2016.

21. Yang S, Xu J, Shao W, Geng C, Li J, Guo F, Miao H, Shen W, Ye T, Liu Y, et al: Radiation-induced bystander effects in A549 cells exposed to $6 \mathrm{MV}$ X-rays. Cell Biochem Biophys 72: 877-882, 2015.

22. Suzuki M: Significance of radiation-induced bystander effects in radiation therapy. Igaku Butsuri 34: 70-78, 2014 (In Japanese).

23. Howe O, O'Sullivan J, Nolan B, Vaughan J, Gorman S, Clarke C, McClean B and Lyng FM: Do radiation-induced bystander effects correlate to the intrinsic radiosensitivity of individuals and have clinical significance? Radiat Res 171: 521-529, 2009. 